\title{
Vibration Control of a Viscoelastic Flexible Marine Riser with Vessel Dynamics
}

\section{Lamia Seghour ${ }^{a}$, Amirouche Berkani $^{b}$, Nasser-eddine Tatar $^{c}$ and Fardin Saedpanah ${ }^{d}$}

${ }^{a}$ Faculty of Mathematics, University of Sciences and Technology Houari Boumediene

BP 32 El Alia, 16111 Bab Ezzouar Algiers, Algeria

${ }^{b}$ Faculty of Mathematics and Informatics, University Mohamed El Bachir El Ibrahimi

Bordj Bou Arréridj, 34030 El-Anasser, Algeria

${ }^{c}$ Department of Mathematics and Statistics, King Fahd University of

Petroleum and Minerals

31261 Dhahran, Saudi Arabia

${ }^{d}$ Department of Mathematics, University of Kurdistan

P.O. Box 416, Sanandaj, Iran

E-mail(corresp.): aberkanid@gmail.com

Received August 3, 2017; revised May 21, 2018; accepted May 23, 2018

\begin{abstract}
In this work, we investigate the asymptotic behavior of solutions of a viscoelastic flexible marine riser with vessel dynamics. Under a suitable control applied at the top end of the riser, we establish explicit decay rates for a large class of relaxation functions. In particular, exponentially and polynomially (or power type) decaying functions are included in this class. Our method is based on the multiplier technique. Numerical simulations justifying the effectiveness of the proposed boundary control to suppress the vibrations of the flexible marine riser are provided.
\end{abstract}

Keywords: stability, vibration control, flexible marine riser, boundary control, EulerBernoulli beam structure, viscoelasticity.

AMS Subject Classification: 35L20; 93D15; 93D20.

\section{Introduction}

When a cylinder is submerged in a moving fluid we assist to the formation of complex vertices which destabilize the cylinder from its initial position. They 
make the cylinder vibrate. This phenomenon is called Vortex-induced vibrations. It is one of the main concern in engineering and in particular in offshore industry. Companies are spending a lot of money in their investments to remedy to this problem. Indeed, vortices produce unwanted and in fact destructive vibrations of the pipeline connecting the well in the bottom of the sea to the floating platform. In addition to these "transversal" vibrations and as the riser transports crude oil or natural gaz there will be internal pressure due to the fluid flow (often with pulses). There are also other causes of vibrations like transmission of waves, earthquake ground motions, etc.

An immediate consequence of these vibrations is the partial or complete destruction of the riser, or at least a limitation of its lifetime. This damage has to be predicated long before. A failure will affect the productivity or lead to an ecological disaster in case of leakage. Therefore there is a need for periodic inspections and maintenance.

Several ways have been devised to improve the efficiency and the lifespan of the riser. In particular, different forms, shapes and material have been used to suppress or at least reduce the unwanted vibrations. Also, some additional devices have been added to the structure like fairings, streaks and tensioner to increase the stiffness or the riser. Yet these devices are not able to give satisfactory results. Researchers have been forced to use sensors and actuators to control the vibrations. Unless the platform is fixed and the riser is clamped, for instance, to the supports (legs) of the structure, it is not practical to place these devices in inaccessible spots. Unfortunately, most of the platforms are floating because of the deep water (up to $3 \mathrm{~km}$ ) and the need for mobility. The best location for control is the top of the riser. That is a boundary control at only one endpoint. This direction of research has gained a lot of attention. A successful boundary control should stabilize the system quickly enough to avoid any damage. We refer the reader to $[4,6,8,13,17,23]$ where the authors studied the boundary control of flexible marine riser. It is worth mentioning the work of Seghour et al. [29] where the authors considered the problem

$$
\rho w_{t t}(x, t)+E I w_{x x x x}(x, t)-E I \int_{0}^{t} h(t-s) w_{x x x x}(x, s) d s-T w_{x x}(x, t)=0,
$$

for all $(x, t) \in(0, L) \times[0, \infty)$, with the boundary conditions and the initial data

$$
\left\{\begin{array}{l}
w(0, t)=w_{x}(0, t)=w_{x x}(L, t)=0, \quad t \geq 0, \\
-E I w_{x x x}(L, t)+E I \int_{0}^{t} h(t-s) w_{x x x}(L, s) d s+T w_{x}(L, t) \\
=u(t)-d_{s} w_{t}(L, t)-M_{s} w_{t t}(L, t), t \geq 0 \\
w(x, 0)=w_{0}(x), \quad w_{t}(x, 0)=w_{1}(x), \quad x \in[0, L] .
\end{array}\right.
$$

Here $w(x, t), w_{t t}(x, t), w(L, t)$ and $w_{t}(L, t)$ are the displacement, acceleration of the riser at the position $x$ for the time $t$, the position and velocity of the vessel at $L$, respectively. The positive constants $\rho, L, E I$ and $T$ represent, the uniform mass per unit length, the length, the bending stiffness and the tension of the riser, respectively. The coefficients $M_{s}$ and $d_{s}$ denote the mass of the surface vessel and the vessel damping, and $u(t)$ is the top boundary control of 
the riser. The integral term in the Equation (1.1) represents the viscoelastic damping term. This term appears in the constitutive relationship between the stress and the strain according to the Boltzmann Principle (see [7]). The kernel $h$ is called the relaxation function. The expressions $w_{0}(x)$ and $w_{1}(x)$ are given initial data. Under the following control

$$
u(t)=\frac{-K}{w_{t}(L, t)}\left[w_{x x x}(L, t)^{2}+w(L, t)^{2}+\left(\int_{0}^{t} h(t-s) w_{x x x}(L, s) d s\right)^{2}\right], t \geq 0
$$

where $K$ is positive constant, for $w_{t}(L, t) \neq 0$. The authors proved an exponential decay result for solutions of the problem (1.1)-(1.2) for kernels $h$ verifying the condition

$$
0<h^{\prime}(t)+\gamma h(t) \leq \xi(t), \quad t \geq 0
$$

for some positive constant $\gamma$ and nonnegative function $\xi(t)$.

Also, in [12] the authors studied a similar problem to (1.1)-(1.2) when $h \equiv 0$ in (1.1) with internal damping of the form $w_{t}(x, t)$.

The boundary control has received great attention in recent years due its numerous applications in engineering such as vibration control of flexible robotic manipulator $[2,3,16]$, the flexible wings of a robotic aircraft [14], the control of a nonuniform gantry crane [11], the industrial moving strip [15], the nonlinear axially moving string [19] and the references therein. The boundary stabilization and control has been developed for the Euler-Bernoulli beam, by among others: Krall [20], de Querioz et al. [5], Li et al. [21], Andrews et al. [1], Guo and Huang [10], Guo and Guo [9] (see also the references therein). In particular, dissipative mechanisms of viscoelastic type have been implemented. Park et al. [28] looked into the following problem

$$
w_{t t}(x, t)+w_{x x x x}(x, t)-\int_{0}^{t} h(t-\tau) w_{x x x x}(x, \tau) d \tau+g\left(w_{t}(x, t)\right)=0,
$$

where $(x, t) \in[0, L] \times(0, \infty)$, with the boundary conditions and initial data

$$
\left\{\begin{array}{l}
w(0, t)=w_{x}(0, t)=w_{x x}(L, t)=w_{x x x}(0, t)=0, \quad t \geq 0, \\
w_{x x x}(L, t)-\int_{0}^{t} h(t-\tau) w_{x x x}(L, t) d \tau=f(y(L, t)), t \geq 0, \\
w(x, 0)=w_{0}(x), w_{t}(x, 0)=w_{1}(x) x \in[0, L] .
\end{array}\right.
$$

They proved the existence of solutions using the Faedo-Galerkin method and investigated the exponential stability of the system by using the multiplier technique for kernels $h$ of an exponential type, i.e.

$$
\exists k_{i}>0, i=0, \ldots, 2:-k_{0} h(t) \leq h_{t}(t) \leq-k_{1} h(t), \quad 0<h_{t t}(t) \leq k_{2} h(t) .
$$

A similar result was obtained by the same authors in [27] together with a boundary output feedback control

$$
\left\{\begin{array}{l}
w(0, t)=w_{x}(0, t)=w_{x x}(L, t)=0, \quad t \geq 0 \\
w_{x x x}(L, t)-\int_{0}^{t} g(t-\tau) w_{x x x}(L, t) d \tau=u(t), t \geq 0 \\
w_{\text {out }}=w_{t}(L, t) \quad t \geq 0
\end{array}\right.
$$


where $w_{\text {out }}$ is the measured signal of the system at time $t$ with

$$
\left\{\begin{array}{l}
u(t)=k(t) w_{t}(L, t), \quad k(0)>0, \quad t \geq 0, \\
k^{\prime}(t)=r w_{t}^{2}(L, t), \quad t \geq 0 .
\end{array}\right.
$$

An analogous result was also established by Kang et al. [18] with the boundary conditions

$$
\left\{\begin{array}{l}
w(0, t)=w_{x}(0, t)=w_{x x}(L, t)=0, \quad t \geq 0 \\
w_{x x x}(L, t)-\int_{0}^{t} g(t-\tau) w_{x x x}(L, t) d \tau=u(t)-\tilde{\theta} \sin (t), t \geq 0 \\
w_{\text {out }}=y_{t}(L, t) \quad t \geq 0
\end{array}\right.
$$

where $\tilde{\theta}$ is a positive constant. For exponentially decaying kernels, using the multiplier technique they proved the exponential stability under the following adaptive output feedback controller

$$
\left\{\begin{array}{l}
u(t)=h(t) w_{t}(L, t)+\theta(t) \sin t, \quad t \geq 0 \\
h_{t}(t)=r w_{t}^{2}(L, t), \quad h(0)=h_{0}>0, \quad t \geq 0, \quad r>0 \\
\theta_{t}(t)=w_{t}(L, t) \sin t, \quad \theta(0)=\theta_{0}
\end{array}\right.
$$

The main objective of this work, is to establish different types of decay rates of the system (1.1)-(1.2) under a suitable control acting on top of the riser. This will be shown for a large class of kernels. Namely, we suppose that $h^{\prime}(t) \leq \xi(t)$ for some non-negative function $\xi(t)$ and the kernel $h$ verifies

$$
h(t-s) \geq \mu(t) \int_{t}^{\infty} h(\sigma-s) d \sigma, t>0
$$

for some function $\mu(t)$. This condition is satisfied by a large class of functions including polynomials and exponentials (see Remark 2). It is important to consider these conditions which allow the use of more materials that could be more convenient. For other types of kernels, one can consult $[2,19]$.

The well-posedness of our system (1.1)-(1.2) can be established by using Faedo-Galerkin method, we refer the reader to [3]. We set

$$
\mathcal{V}=\left\{w \in H^{2}(0, L), w(0)=w_{x}(0)=0\right\}, \quad \mathcal{W}=\left\{w \in H^{4}(0, L), w_{x x}(L)=0\right\},
$$

where $H^{2}(0, L)$ and $H^{4}(0, L)$ are the usual Sobolev spaces.

Proposition 1. Let $w_{0} \in \mathcal{W}, w_{1} \in \mathcal{V}$ and $h(t)$ be a nonnegative summable kernel. Under the control $u(t)$ defined in (3.1), the system (1.1)-(1.2) has a unique solution $w$ in the class

$$
w \in L^{\infty}([0, T) ; \mathcal{W}), w_{t} \in L^{\infty}([0, T) ; \mathcal{V}), w_{t t} \in L^{\infty}\left([0, T) ; L^{2}(0, L)\right),
$$

where $T>0$.

The rest of our paper is organized as follows. In Section 2 we prepare some lemmas which will be useful in the proof of our result. The arbitrary decay result under a suitable boundary control $u(t)$ is stated and proved in Section 3. Simulations are presented to illustrate the performance of the proposed control in Section 4, and some conclusions are given in Section 5. 


\section{Preliminaries results}

In this section, we give some lemmas which will be used throughout this paper. First, to simplify the notation, we denote by $\square$ the binary operator, defined by

$$
(h \square v)(t)=\int_{0}^{L} \int_{0}^{t} h(t-s)(v(t)-v(s))^{2} d s d x, \quad t \geq 0 .
$$

We shall use the following inequalities.

Lemma 1. Under our boundary conditions (1.2), we have

$$
w^{2}(x, t) \leq L\left\|w_{x}\right\|_{2}^{2}, \quad w^{2}(x, t) \leq L^{3}\left\|w_{x x}\right\|_{2}^{2}, \quad w_{x}^{2}(x, t) \leq L\left\|w_{x x}\right\|_{2}^{2},
$$

for all $(x, t) \in[0, L] \times \mathbb{R}^{+}$and

$$
\|w\|_{2}^{2} \leq L^{2}\left\|w_{x}\right\|_{2}^{2} \leq L^{4}\left\|w_{x x}\right\|_{2}^{2} \quad \forall x \in[0, L] .
$$

To preserve the hyperbolicity of our system (1.1)-(1.2), we assume that the kernel $h$ satisfies

$$
1-\int_{0}^{\infty} h(s) d s=: 1-\kappa>0 .
$$

Lemma 2. The energy functional $\mathcal{E}(t)$ of the problem (1.1)-(1.2) given by

$$
\begin{aligned}
2 \mathcal{E}(t)= & \rho\left\|w_{t}\right\|_{2}^{2}+E I\left(1-\int_{0}^{t} h(s) d_{s}\right)\left\|w_{x x}\right\|_{2}^{2}+T\left\|w_{x}\right\|_{2}^{2} \\
& +M_{s} w_{t}^{2}(L, t)+E I\left(h \square w_{x x}\right),
\end{aligned}
$$

satisfies, for all $t \geq 0$

$$
\frac{d}{d t} \mathcal{E}(t)=-\frac{E I}{2} h(t)\left\|w_{x x}\right\|_{2}^{2}+\frac{E I}{2}\left(h^{\prime} \square w_{x x}\right)-d_{s} w_{t}^{2}(L, t)+u(t) w_{t}(L, t),
$$

where $\|.\|_{2}$ is the norm in $L^{2}(0, L)$.

Proof. By multiplying the Equation (1.1) by $w_{t}$, integrating over $(0, L)$ and using the boundary conditions (1.2), we get

$$
\begin{aligned}
& \frac{1}{2} \frac{d}{d t}\left(\rho\left\|w_{t}\right\|_{2}^{2}+E I\left\|w_{x x}\right\|_{2}^{2}+T\left\|w_{x}\right\|_{2}^{2}+M_{s} w_{t}^{2}(L, t)\right) \\
& \quad=E I \int_{0}^{L} w_{x x t} \int_{0}^{t} h(t-s) w_{x x}(s) d s d x-d_{s} w_{t}^{2}(L, t)+u(t) w_{t}(L, t), \quad t \geq 0 .
\end{aligned}
$$

Now, it suffices to observe that

$$
\begin{gathered}
2 \int_{0}^{L} \omega_{x x t} \int_{0}^{t} h(t-s) \omega_{x x}(s) d s d x=\int_{0}^{L}\left(h^{\prime} \square w_{x x}\right) d x-h(t)\left\|w_{x x}\right\|_{2}^{2} \\
-\frac{d}{d t}\left[\int_{0}^{L}\left(h \square w_{x x}\right) d x-\left(\int_{0}^{t} h(s) d s\right)\left\|w_{x x}\right\|_{2}^{2}\right],
\end{gathered}
$$


for all $t \geq 0$, to conclude.

From this lemma we see that it is not clear that the energy $\mathcal{E}(t)$ of our system $(1.1)-(1.2)$ is decreasing. Now, we set

$$
V(t)=\mathcal{E}(t)+\sum_{i=1}^{4} \lambda_{i} V_{i}(t), \quad t \geq 0,
$$

where $\lambda_{i}, i=1, \ldots, 4$ are positive constants which will be chosen later, and

$$
\begin{aligned}
V_{1}(t) & =\rho \int_{0}^{L} w_{t} w d x+M_{s} w_{t}(L, t) w(L, t), \quad t \geq 0 \\
V_{2}(t) & =-\rho \int_{0}^{L} w_{t} \int_{0}^{t} h(t-s)(w(t)-w(s)) d s d x \\
& -M_{s} w_{t}(L, t) \int_{0}^{t} h(t-s)(w(L, t)-w(L, s)) d s, \quad t \geq 0, \\
V_{3}(t) & =\int_{0}^{t}\left(\int_{t}^{\infty} h(\sigma-s) d \sigma\right)\left\|w_{x x}(s)\right\|_{2}^{2} d s, \quad t \geq 0 \\
V_{4}(t) & =\int_{0}^{t}\left(\int_{t}^{\infty} \xi(\sigma-s) d \sigma\right)\left\|w_{x x}(s)\right\|_{2}^{2} d s, \quad t \geq 0 .
\end{aligned}
$$

Remark 1 . The functionals $V_{i}, i=1, \ldots, 4$ should be chosen in such a way that their derivatives will provide us with similar terms to the ones in the energy expression (kinetic, potential ...) but with negative coefficients. We will control these terms and have the energy with a negative sign in the right hand side of the estimate of the derivative of (2.2).

The first result tells us that $V(t)$ and $\mathcal{E}(t)+V_{3}(t)+V_{4}(t)$ are equivalent.

Lemma 3. There exist $\rho_{i}>0, i=1,2$ such that

$$
\rho_{1}\left[\mathcal{E}(t)+V_{3}(t)+V_{4}(t)\right] \leq V(t) \leq \rho_{2}\left[\mathcal{E}(t)+V_{3}(t)+V_{4}(t)\right],
$$

for all $t \geq 0$.

Proof. By Young's and Hölder's inequalities, we obtain

$$
V_{1}(t) \leq \frac{\rho}{2}\left\|w_{t}\right\|_{2}^{2}+\frac{\rho L^{4}}{2}\left\|w_{x x}\right\|_{2}^{2}+\frac{M_{s}}{2} w^{2}(L, t)+\frac{M_{s}}{2} w_{t}^{2}(L, t), t \geq 0 .
$$

Therefore, by using Lemma 1, we get

$$
V_{1}(t) \leq \frac{\rho}{2}\left\|w_{t}\right\|_{2}^{2}+\left(\rho L+M_{s}\right) \frac{L^{3}}{2}\left\|w_{x x}\right\|_{2}^{2}+\frac{M_{s}}{2} w_{t}^{2}(L, t), t \geq 0 .
$$

For $V_{2}(t)$, it easy to see

$$
\begin{aligned}
V_{2}(t) & \leq \frac{\rho}{2}\left\|w_{t}\right\|_{2}^{2}+\frac{\rho L^{4} \kappa}{2}\left(h \square w_{x x}\right)+\frac{M_{s}}{2} w_{t}^{2}(L, t) \\
& +\frac{M_{s} \kappa}{2} \int_{0}^{t} h(t-s)(w(L, t)-w(L, s))^{2} d s, t \geq 0 .
\end{aligned}
$$


Using Lemma 1 again, we have

$$
\int_{0}^{t} h(t-s)(w(L, s)-w(L, t))^{2} d s \leq L^{3}\left(h \square w_{x x}\right), t \geq 0 .
$$

The last relation, imply that

$$
V_{2}(t) \leq \frac{\rho}{2}\left\|w_{t}\right\|_{2}^{2}+\left(\rho L+M_{s}\right) \frac{\kappa L^{3}}{2}\left(h \square w_{x x}\right)+\frac{M_{s}}{2} w_{t}^{2}(L, t), t \geq 0 .
$$

Gathering (2.3) and (2.4), we end up with

$$
\begin{aligned}
V(t) \leq & \frac{\rho}{2}\left(1+\lambda_{1}+\lambda_{2}\right)\left\|w_{t}\right\|_{2}^{2}+\frac{1}{2}\left[E I+\lambda_{2}\left(\rho L+M_{s}\right) \kappa L^{3}\right]\left(h \square w_{x x}\right)(t) \\
& +\frac{1}{2}\left[E I\left(1-\int_{0}^{t} h(s) d s\right)+\lambda_{1}\left(\rho L+M_{s}\right) L^{3}\right]\left\|w_{x x}\right\|_{2}^{2} \\
& +\frac{T}{2}\left\|w_{x}\right\|_{2}^{2}+\frac{1}{2} M_{s}\left(1+\lambda_{1}+\lambda_{2}\right) w_{t}^{2}(L, t)+\lambda_{3} V_{3}(t)+\lambda_{4} V_{4}(t), t \geq 0 .
\end{aligned}
$$

Therefore,

$$
V(t) \leq \rho_{2}\left(\mathcal{E}(t)+\sum_{j=3}^{4} V_{j}(t)\right), t \geq 0
$$

for some positive constant $\rho_{2}$. Similarly,

$$
\begin{aligned}
2 V(t) \leq & \rho\left(1-\lambda_{1}-\lambda_{2}\right)\left\|w_{t}\right\|_{2}^{2}+\left[E I-\lambda_{2}\left(\rho L+M_{s}\right) \kappa L^{3}\right]\left(h \square w_{x x}\right) \\
& +\left[E I(1-\kappa)-\lambda_{1}\left(\rho L+M_{s}\right) L^{3}\right]\left\|w_{x x}\right\|_{2}^{2}+T\left\|w_{x}\right\|_{2}^{2} \\
& +M_{s}\left(1-\lambda_{1}-\lambda_{2}\right) w_{t}^{2}(L, t)+2 \lambda_{3} V_{3}(t)+2 \lambda_{4} V_{4}(t), t \geq 0 .
\end{aligned}
$$

Hence,

$$
V(t) \geq \rho_{2}\left(\mathcal{E}(t)+\sum_{j=3}^{4} V_{j}(t)\right), \quad t \geq 0
$$

for some constant $\rho_{1}>0$, provided that

$$
\lambda_{1}<\min \left[1, \frac{E I(1-\kappa)}{\left(\rho L+M_{s}\right) L^{3}}\right], \lambda_{2}<\min \left[1-\lambda_{1}, \frac{E I}{\left(\rho L+M_{s}\right) \kappa L^{3}}\right] .
$$

The following inequality will be used repeatedly in the sequel.

Lemma 4. We have

$$
\delta \gamma \leq \eta \delta^{2}+\frac{\gamma^{2}}{4 \eta}, \quad \delta, \gamma \in \mathbb{R}, \quad \eta>0
$$

The identity to follow is easy to justify and is helpful to prove our results. 
Lemma 5. (See [30]) We have for $h \in C(0, \infty)$ and $y \in C\left((0, \infty) ; L^{2}(0, L)\right)$

$$
\begin{aligned}
\int_{0}^{L} y \int_{0}^{t} h(t-s) y(s) d s d x= & \frac{1}{2}\left(\int_{0}^{t} h(s) d s\right)\|y\|_{2}^{2}-\frac{1}{2}(h \square y)(t) \\
& +\frac{1}{2} \int_{0}^{t} h(t-s)\|y(s)\|_{2}^{2} d s
\end{aligned}
$$

for all $t \geq 0$.

\section{Asymptotic behavior}

Our goal in this section is to prove a stabilization result. We start by giving some notions and assumptions used in this work in line with [30]. Let $\mathcal{A}$ be a measurable set, such that $\mathcal{A} \subset \mathbb{R}^{+}$, we denote by $\hat{h}(\mathcal{A})$ the probability measure

$$
\hat{h}(\mathcal{A})=\frac{1}{\kappa} \int_{\mathcal{A}} h(s) d s .
$$

The non-decreasingness set of $h$ is defined by $Q_{h}:=\left\{s \in \mathbb{R}^{+}: h^{\prime}(s) \geq 0\right\}$, we also define the non-decreasingness rate of $h$ by $\mathcal{R}_{h}:=\hat{h}\left(Q_{h}\right)$. We suppose that the kernel $h(t)$ verifies (see [30]):

$$
h(t) \geq 0 \text { for all } t \geq 0 \text { and } 0<\kappa=\int_{0}^{+\infty} h(s) d s<1 .
$$

(H2) $\quad h(t)$ is absolutely continuous and of bounded variation on $(0, \infty)$ and $h^{\prime}(t) \leq \xi(t)$ for some non-negative summable function $\xi(t)\left(=\max \left\{0, h^{\prime}(t)\right\}\right)$ and almost all $t>0$.

(H3) There exist two functions $\mu_{i}(t)>0, i=1,2$ such that

$$
\begin{aligned}
& h(t-s) \geq \mu_{1}(t) \int_{t}^{\infty} h(\sigma-s) d \sigma, t>0, \\
& \xi(t-s) \geq \mu_{2}(t) \int_{t}^{\infty} \xi(\sigma-s) d \sigma, t>0 .
\end{aligned}
$$

Remark 2. Notice that this assumption (H3) is satisfied by a large class of functions namely functions of polynomial type and functions of exponential type. Indeed, $\xi(t)=(1+t)^{-\alpha}, \alpha>0$ satisfies the assumption with $\mu(t)=$ $(\alpha-1) /(1+t)$. The function $\xi(t)=e^{-\beta t}, \beta>0$ satisfies the assumption with $\mu(t)=\beta$.

Let $t_{*}>0$ be a number such that $\int_{0}^{t_{*}} h(s) d s=h_{*}>0$. For simplicity, we consider kernels continuous everywhere and continuously differentiable a.e.

In order to stabilize the system (1.1)-(1.2), we propose the following boundary control

$$
u(t)=d_{s} w_{t}(L, t), \quad t \geq 0 .
$$


Remark 3. The boundary control law defined in (3.1), is proposed to suppress the vibrations of the flexible marine riser in a fast manner. There are other ways how to design suitable boundary controllers for PDEs in the literature, the reader is referred to $[22,24,25,26]$, and their references.

Now, we are ready to state and prove our main result.

Theorem 1. Under the assumptions (H1)-(H3) and the control $u(t)$ defined in (3.1), if $\mathcal{R}_{h}$ is sufficiently small, then, for small $L^{1}$-norm of $\xi$ there exist positive constants $\Lambda$ and $\nu$ such that

(a) if $\lim _{t \rightarrow \infty} \mu_{1}(t)=0$ or $\lim _{t \rightarrow \infty} \mu_{2}(t)=0$,

$$
\mathcal{E}(t) \leq \Lambda \exp \left(-\nu \int_{0}^{t} \min \left\{\mu_{1}(s), \mu_{2}(s)\right\} d s\right), t \geq 0 .
$$

(b) If $\lim _{t \rightarrow \infty} \mu_{1}(t) \neq 0$ and $\lim _{t \rightarrow \infty} \mu_{2}(t) \neq 0$, we have

$$
\mathcal{E}(t) \leq \Lambda e^{-\nu t}, t \geq 0 \text {. }
$$

Proof. A differentiation of $V_{1}(t)$ with respect to $t$ along solutions of (1.1) gives

$$
\begin{aligned}
\frac{d}{d t} V_{1}(t)= & \rho\left\|w_{t}\right\|_{2}^{2}+M_{s} w_{t}(L, t)^{2}+M_{s} w_{t t}(L, t) w(L, t) \\
& +I_{1}+I_{2}+I_{3}, \quad t \geq 0,
\end{aligned}
$$

where

$$
\begin{aligned}
& I_{1}=-E I \int_{0}^{L} w w_{x x x x} d x, \quad I_{2}=E I \int_{0}^{L} w \int_{0}^{t} h(t-s) w_{x x x x}(s) d s d x, \\
& I_{3}=T \int_{0}^{L} w w_{x x} d x .
\end{aligned}
$$

Integrating by parts in $I_{i} i=1, \ldots, 3$ and using the boundary conditions (1.2), we get

$$
\begin{aligned}
I_{1}= & -E I w(L, t) w_{x x x}(L, t)-E I\left\|w_{x x}\right\|_{2}^{2}, \quad t \geq 0, \\
I_{2}= & E I w(L, t) \int_{0}^{t} h(t-s) w_{x x x}(L, s) d s \\
& +E I \int_{0}^{L} w_{x x} \int_{0}^{t} h(t-s) w_{x x}(s) d s d x, \quad t \geq 0, \\
I_{3}= & T w_{x}(L, t) w(L, t)-T\left\|w_{x}\right\|_{2}^{2}, \quad t \geq 0 .
\end{aligned}
$$

Substituting the estimates (3.3)-(3.5) in Equation (3.2), taking into account the boundary conditions (1.2), the expression of the control $u(t)$ and canceling common terms, we obtain

$$
\begin{aligned}
\frac{d}{d t} V_{1}(t)= & \rho\left\|w_{t}\right\|_{2}^{2}+M_{s} w_{t}^{2}(L, t)-E I\left\|w_{x x}\right\|_{2}^{2}-T\left\|w_{x}\right\|_{2}^{2} \\
& +E I \int_{0}^{L} w_{x x} \int_{0}^{t} h(t-s) w_{x x}(s) d s d x, \quad t \geq 0
\end{aligned}
$$


and by the identity (2.5) in Lemma 5 we get

$$
\begin{aligned}
\frac{d}{d t} V_{1}(t) & \leq \rho\left\|w_{t}\right\|_{2}^{2}+M_{s} w_{t}^{2}(L, t)-E I\left(1-\frac{\kappa}{2}\right)\left\|w_{x x}\right\|_{2}^{2}-T\left\|w_{x}\right\|_{2}^{2} \\
& -\frac{E I}{2}\left(h \square w_{x x}\right)+\frac{E I}{2} \int_{0}^{t} h(t-s)\left\|w_{x x}(s)\right\|_{2}^{2} d s, \quad t \geq 0 .
\end{aligned}
$$

For the functional $V_{2}(t)$, we have

$$
\begin{aligned}
& \frac{d}{d t} V_{2}(t)=-\rho\left(\int_{0}^{t} h(s) d s\right)\left\|w_{t}\right\|_{2}^{2}-M_{s}\left(\int_{0}^{t} h(s) d s\right) w_{t}^{2}(L, t) \\
& \quad-\rho \int_{0}^{L} w_{t} \int_{0}^{t} h^{\prime}(t-s)(w(t)-w(s)) d s d x-M_{s} w_{t}(L, t) \\
& \left.\quad \times \int_{0}^{t} h^{\prime}(t-s)(w(L, t)-w(L, s)) d s-M_{s} w_{t t} L, t\right) \\
& \quad \times \int_{0}^{t} h(t-s)(w(L, t)-w(L, s)) d s+I_{4}+I_{5}+I_{6}+I_{7}, t \geq 0
\end{aligned}
$$

where

$$
\begin{aligned}
& I_{4}=E I \int_{0}^{L} w_{x x x x} \int_{0}^{t} h(t-s)(w(t)-w(s)) d s d x \\
& I_{5}=-E I \int_{0}^{L}\left(\int_{0}^{t} h(t-s) w_{x x x x}(s) d s\right) \int_{0}^{t} h(t-s)(w(t)-w(s)) d s d x \\
& I_{6}=-T \int_{0}^{L} w_{x x} \int_{0}^{t} h(t-s)(w(t)-w(s)) d s d x .
\end{aligned}
$$

An integration by parts of $I_{i}, i=4,5,6$ and using our boundary conditions (1.2), we obtain for all $t \geq 0$,

$$
\begin{aligned}
I_{4}= & E I w_{x x x}(L, t) \int_{0}^{t} h(t-s)(w(L, t)-w(L, s)) d s \\
& +E I \int_{0}^{L} w_{x x} \int_{0}^{t} h(t-s)\left(w_{x x}(t)-w_{x x}(s)\right) d s d x, \\
I_{5}= & -E I\left(\int_{0}^{t} h(t-s) w_{x x x}(L, s) d s\right) \int_{0}^{t} h(t-s)(w(L, t)-w(L, s)) d s \\
& -E I \int_{0}^{L}\left(\int_{0}^{t} h(t-s) w_{x x}(s) d s\right) \int_{0}^{t} h(t-s)\left(w_{x x}(t)-w_{x x}(s)\right) d s d x
\end{aligned}
$$

and

$$
\begin{aligned}
I_{6}= & -T w_{x}(L, t) \int_{0}^{t} h(t-s)(w(L, t)-w(L, s)) d s \\
& +T \int_{0}^{L} w_{x} \int_{0}^{t} h(t-s)\left(w_{x}(t)-w_{x}(s)\right) d s d x .
\end{aligned}
$$


Collecting the estimates (3.8)-(3.10) in (3.7), using again the boundary conditions, the expression of the control $u(t)$ after canceling common terms, we see that

$$
\begin{aligned}
& \frac{d}{d t} V_{2}(t)=-\left(\int_{0}^{t} h(s) d s\right)\left(\rho\left\|w_{t}\right\|_{2}^{2}+M_{s} w_{t}^{2}(L, t)\right) \\
& -\rho \int_{0}^{L} w_{t} \int_{0}^{t} h^{\prime}(t-s)(w(t)-w(s)) d s d x-M_{s} w_{t}(L, t) \int_{0}^{t} h^{\prime}(t-s) \\
& \times(w(L, t)-w(L, s)) d s+E I \int_{0}^{L}\left|\int_{0}^{t} h(t-s)\left(w_{x x}(t)-w_{x x}(s)\right) d s\right|^{2} d x \\
& +T \int_{0}^{L} w_{x} \int_{0}^{t} h(t-s)\left(w_{x}(t)-w_{x}(s)\right) d s d x+E I\left(1-\int_{0}^{t} h(s) d s\right) \\
& \quad \times \int_{0}^{L} w_{x x} \int_{0}^{t} h(t-s)\left(w_{x x}(t)-w_{x x}(s)\right) d s d x, \quad t \geq 0 .
\end{aligned}
$$

Now, we estimate the terms in (3.11). For all measurable sets $\mathcal{A}$ and $Q$ such that $\mathcal{A}=\mathbb{R}^{+} \backslash Q$, we start by the last term

$$
\begin{aligned}
\int_{0}^{L} & w_{x x} \int_{0}^{t} h(t-s)\left(w_{x x}(t)-w_{x x}(s)\right) d s d x \\
\quad & \int_{0}^{L} w_{x x} \int_{\mathcal{A} \cap[0, t]} h(t-s)\left(w_{x x}(t)-w_{x x}(s)\right) d s d x \\
& +\int_{0}^{L} w_{x x} \int_{Q \cap[0, t]} h(t-s)\left(w_{x x}(t)-w_{x x}(s)\right) d s d x \\
& \leq \int_{0}^{L} w_{x x} \int_{\mathcal{A} \cap[0, t]} h(t-s)\left(w_{x x}(t)-w_{x x}(s)\right) d s d x \\
+ & \left(\int_{Q \cap[0, t]} h(t-s) d s\right)\left\|w_{x x}\right\|_{2}^{2}-\int_{0}^{L} w_{x x} \int_{Q \cap[0, t]} h(t-s) w_{x x}(s) d s d x
\end{aligned}
$$

for all $t \geq 0$. Clearly, for $\eta_{1}>0$, we have

$$
\begin{aligned}
& \int_{0}^{L} w_{x x} \int_{\mathcal{A}_{t}} h(t-s)\left(w_{x x}(t)-w_{x x}(s)\right) d s d x \\
& \quad \leq \eta_{1}\left\|w_{x x}\right\|_{2}^{2}+\frac{\kappa}{4 \eta_{1}} \int_{0}^{L} \int_{\mathcal{A}_{t}} h(t-s)\left(w_{x x}(t)-w_{x x}(s)\right)^{2} d s d x
\end{aligned}
$$

where we have used the notation $\mathcal{B}_{t}=\mathcal{B} \cap[0, t]$ and

$$
\begin{aligned}
& \int_{0}^{L} w_{x x} \int_{Q_{t}} h(t-s) w_{x x}(s) d s d x \\
& \quad \leq \frac{1}{2}\left(\int_{Q_{t}} h(t-s) d s\right)\left\|w_{x x}\right\|_{2}^{2}+\frac{1}{2} \int_{Q_{t}} h(t-s)\left\|w_{x x}(s)\right\|_{2}^{2} d s, \quad t \geq 0 .
\end{aligned}
$$


These two estimations show that (3.12), becomes

$$
\begin{aligned}
\int_{0}^{L} & w_{x x} \int_{0}^{t} h(t-s)\left(w_{x x}(t)-w_{x x}(s)\right) d s d x \leq\left(\eta_{1}+\frac{3}{2} \int_{Q_{t}} h(t-s) d s\right) \\
& \times\left\|w_{x x}\right\|_{2}^{2}+\frac{\kappa}{4 \eta_{1}} \int_{0}^{L} \int_{\mathcal{A}_{t}} h(t-s)\left(w_{x x}(t)-w_{x x}(s)\right)^{2} d s d x \\
& +\frac{1}{2} \int_{Q_{t}} h(t-s)\left\|w_{x x}(s)\right\|_{2}^{2} d s, \quad \eta_{1}>0, \quad t \geq 0
\end{aligned}
$$

For the 5 th term, it holds that for $\eta_{2}>0$

$$
\begin{aligned}
\int_{0}^{L} & \left|\int_{0}^{t} h(t-s)\left(w_{x x}(t)-w_{x x}(s)\right) d s\right|^{2} d x \leq\left(1+\frac{1}{\eta_{2}}\right) \kappa \\
& \times \int_{0}^{L} \int_{\mathcal{A}_{t}} h(t-s)\left(w_{x x}(t)-w_{x x}(s)\right)^{2} d s d x+\left(1+\eta_{2}\right)\left(\int_{Q_{t}} h(t-s) d s\right) \\
& \times \int_{0}^{L} \int_{Q_{t}} h(t-s)\left(w_{x x}(t)-w_{x x}(s)\right)^{2} d s d x, \quad t \geq 0
\end{aligned}
$$

For the 6 th term, using Lemma 4 , it is easy to see that

$$
\begin{aligned}
& \int_{0}^{L} w_{x} \int_{0}^{t} h(t-s)\left(w_{x}(t)-w_{x}(s)\right) d s d x \leq \eta_{3}\left\|w_{x}\right\|_{2}^{2}+\frac{\kappa L}{2 \eta_{3}} \\
& \quad \times \int_{0}^{L} \int_{\mathcal{A}_{t}} h(t-s)\left(w_{x x}(t)-w_{x x}(s)\right)^{2} d s d x+\frac{\kappa L}{2 \eta_{3}}\left(\int_{Q_{t}} h(t-s) d s\right) \\
& \quad \times \int_{0}^{L} \int_{Q_{t}} h(t-s)\left(w_{x x}(t)-w_{x x}(s)\right)^{2} d s d x, \quad \eta_{3}>t \geq 0 .
\end{aligned}
$$

Using Lemma 4 again and the assumption (H2), the third and the forth terms in the right-hand side of $(3.12)$ can be estimated for $\eta_{4}>0$ as follows

$$
\begin{aligned}
& \int_{0}^{L} w_{t} \int_{0}^{t} h^{\prime}(t-s)(w(t)-w(s)) d x d s \\
& \quad \leq \eta_{4}\left\|w_{t}\right\|_{2}^{2}+\frac{L^{4}}{4 \eta_{4}}\left(\int_{0}^{t}\left|h^{\prime}(s)\right| d s\right)\left(\left|h^{\prime}\right| \square w_{x x}\right) \\
& \quad \leq \eta_{4}\left\|w_{t}\right\|_{2}^{2}-\frac{L^{4}}{4 \eta_{4}} B V[h] \int_{0}^{L} \int_{\mathcal{A}_{t}} h^{\prime}(t-s)\left(w_{x x}(t)-w_{x x}(s)\right)^{2} d s d x \\
& \quad+\frac{L^{4}}{4 \eta_{4}}\left(\int_{Q_{t}} \xi(t-s) d s\right) \int_{0}^{L} \int_{Q_{t}} \xi(t-s)\left(w_{x x}(t)-w_{x x}(s)\right)^{2} d s d x, t \geq 0
\end{aligned}
$$

and

$$
\begin{gathered}
w_{t}(L, t) \int_{0}^{t} h^{\prime}(t-s)(w(L, t)-w(L, s)) d s \leq \eta_{4} w_{t}^{2}(L, t)+\frac{1}{4 \eta_{4}}\left(\int_{0}^{t}\left|h^{\prime}(s)\right| d s\right) \\
\times \int_{0}^{t}\left|h^{\prime}(t-s)\right|(w(L, t)-w(L, s))^{2} d s \leq \eta_{4} w_{t}^{2}(L, t)-\frac{L^{3}}{4 \eta_{4}} B V[h]
\end{gathered}
$$




$$
\begin{aligned}
& \times \int_{0}^{L} \int_{\mathcal{A}_{t}} h^{\prime}(t-s)\left(w_{x x}(t)-w_{x x}(s)\right)^{2} d s d x+\frac{L^{3}}{4 \eta_{4}}\left(\int_{Q_{t}} \xi(t-s) d s\right) \\
& \times \int_{0}^{L} \int_{Q_{t}} \xi(t-s)\left(w_{x x}(t)-w_{x x}(s)\right)^{2} d s d x, \quad t \geq 0,
\end{aligned}
$$

where $B V[h]$ is the total variation of $h$.

The insertion of (3.13)-(3.17) into Equation (3.11) gives for all $t \geq t_{*}>0$

$$
\begin{aligned}
& \frac{d}{d t} V_{2}(t) \leq\left(\eta_{4}-h_{*}\right)\left(\rho\left\|w_{t}\right\|_{2}^{2}+M_{s} w_{t}^{2}(L, t)\right)+T \eta_{3}\left\|w_{x}\right\|_{2}^{2}+\frac{E I}{2}\left(1-h_{*}\right) \\
& \times \int_{Q_{t}} h(t-s)\left\|w_{x x}(s)\right\|_{2}^{2} d s+E I\left(1-h_{*}\right)\left(\eta_{1}+\frac{3}{2} \int_{Q_{t}} h(t-s) d s\right)\left\|w_{x x}\right\|_{2}^{2} \\
& +\left[E I\left(1+\frac{1-h_{*}}{4 \eta_{1}}+\frac{1}{\eta_{2}}\right)+\frac{T L}{2 \eta_{3}}\right] \kappa \int_{0}^{L} \int_{\mathcal{A}_{t}} h(t-s)\left(w_{x x}(s)-w_{x x}(t)\right)^{2} d s d x \\
& +\left[E I\left(1+\eta_{2}\right)+\frac{L}{2 \eta_{3}}\right]\left(\int_{Q_{t}} h(t-s) d s\right) \\
& \times \int_{0}^{L} \int_{Q_{t}} h(t-s)\left(w_{x x}(s)-w_{x x}(t)\right)^{2} d s d x+\frac{L^{3}\left(\rho L+M_{s}\right)}{4 \eta_{4}} \\
& \times\left(\int_{Q_{t}} \xi(t-s) d s\right) \int_{0}^{L} \int_{Q_{t}} \xi(t-s)\left(w_{x x}(s)-w_{x x}(t)\right)^{2} d s d x \\
& -\frac{L^{3}\left(\rho L+M_{s}\right)}{4 \eta_{4}} B V[h] \int_{0}^{L} \int_{\mathcal{A}_{t}} h^{\prime}(t-s)\left(w_{x x}(s)-w_{x x}(t)\right)^{2} d s d x .
\end{aligned}
$$

Next, by using assumption (H3) the differentiation of $V_{3}(t)$ and $V_{4}(t)$ (see [30]), yields

$$
\frac{d}{d t} V_{3}(t) \leq \kappa\left\|w_{x x}\right\|_{2}^{2}-\eta_{5} \int_{0}^{t} h(t-s)\left\|w_{x x}(s)\right\|_{2}^{2} d s-\left(1-\eta_{5}\right) \mu_{1}(t) V_{3}(t)
$$

and

$$
\begin{aligned}
\frac{d}{d t} V_{4}(t) \leq & \left(\int_{0}^{\infty} \xi(\sigma) d \sigma\right)\left\|w_{x x}\right\|_{2}^{2}-\eta_{6} \int_{0}^{t} \xi(t-s)\left\|w_{x x}(s)\right\|_{2}^{2} d s \\
& -\left(1-\eta_{6}\right) \mu_{2}(t) V_{4}(t), \quad t \geq 0
\end{aligned}
$$

for $0<\eta_{5}, \eta_{6}<1$. Taking into account the previous estimates (3.6), (3.18), (3.19) and (3.20), and substituting (3.1) in (2.1), we obtain for $t \geq t_{*}>0$

$$
\begin{aligned}
& \frac{d}{d t} V(t) \leq\left[\frac{E I}{2}-\lambda_{2} \frac{L^{3}\left(\rho L+M_{s}\right)}{4 \eta_{4}} B V[h]\right] \\
& \quad \times \int_{0}^{L} \int_{\mathcal{A}_{t}} h^{\prime}(t-s)\left(w_{x x}(s)-w_{x x}(t)\right)^{2} d s d x-\lambda_{1} \frac{E I}{2}\left(h \square w_{x x}\right)
\end{aligned}
$$




$$
\begin{aligned}
+ & {\left[\frac{E I}{2}+\lambda_{2} \frac{L^{3}\left(\rho L+M_{s}\right)}{4 \eta_{4}}\left(\int_{Q_{t}} \xi(t-s) d s\right)\right] \int_{0}^{L} \int_{Q_{t}} \xi(t-s) } \\
& \times\left(w_{x x}(s)-w_{x x}(t)\right)^{2} d s d x+\left[\lambda_{1}+\lambda_{2}\left(\eta_{4}-h_{*}\right)\right]\left(\rho\left\|w_{t}\right\|_{2}^{2}+M_{s} w_{t}^{2}(L, t)\right) \\
+ & {\left[\lambda_{2} E I\left(1-h_{*}\right)\left(\eta_{1}+\frac{3}{2} \int_{Q_{t}} h(t-s) d s\right)+\lambda_{3} \kappa+\left(\lambda_{2} \eta_{3}-\lambda_{1}\right) T\left\|w_{x}\right\|_{2}^{2}\right.} \\
+ & \left.\lambda_{4} \int_{0}^{\infty} \xi(\sigma) d \sigma-\lambda_{1} E I\left(1-\frac{\kappa}{2}\right)\right]\left\|w_{x x}\right\|_{2}^{2}+\lambda_{2}\left[E I\left(1+\frac{1-h_{*}}{4 \eta_{1}}+\frac{1}{\eta_{2}}\right)+\frac{T L}{2 \eta_{3}}\right] \\
& \times \kappa \int_{0}^{L} \int_{\mathcal{A}_{t}} h(t-s)\left(w_{x x}(s)-w_{x x}(t)\right)^{2} d s d x \\
- & \lambda_{4} \eta_{6} \int_{0}^{t} \xi(t-s)\left\|w_{x x}(s)\right\|_{2}^{2} d s+\lambda_{2}\left[E I\left(1+\eta_{2}\right)+\frac{L}{2 \eta_{3}}\right]\left(\int_{Q_{t}} h(t-s) d s\right) \\
& \times \int_{0}^{L} \int_{Q_{t}}^{L} h(t-s)\left(w_{x x}(s)-w_{x x}(t)\right)^{2} d s d x+\left(\frac{\lambda_{1} E I}{2}-\lambda_{3} \eta_{5}\right) \\
& \times \int_{0}^{t} h(t-s)\left\|w_{x x}(s)\right\|_{2}^{2} d s-\lambda_{3}\left(1-\eta_{5}\right) \mu_{1}(t) V_{3}(t) \\
+ & \frac{\lambda_{2} E I}{2}\left(1-h_{*}\right) \int_{Q_{t}} h(t-s)\left\|w_{x x}(s)\right\|_{2}^{2} d s-\lambda_{4}\left(1-\eta_{6}\right) \mu_{2}(t) V_{4}(t) . \quad(3.21)
\end{aligned}
$$

As in [30], we introduce the sets

$$
\begin{aligned}
& \mathcal{A}_{n}:=\left\{s \in \mathbb{R}^{+}, n h^{\prime}(s)+h(s) \leq 0\right\}, n \in \mathbb{N}, \\
& \widetilde{\mathcal{A}}_{n t}=\left\{s \in \mathbb{R}^{+}, 0 \leq s \leq t, n h^{\prime}(t-s)+h(t-s) \leq 0\right\}, n \in \mathbb{N}, \\
& \widetilde{Q}_{h t}=\left\{s \in \mathbb{R}^{+}, 0 \leq s \leq t, 0 \leq h^{\prime}(t-s) \leq \xi(t-s)\right\} .
\end{aligned}
$$

We notice that $\bigcup_{n} \mathcal{A}_{n}=\mathbb{R}^{+} \backslash\left\{Q_{h} \cup \mathcal{N}_{h}\right\}$, where

$$
Q_{h}=\left\{s \in \mathbb{R}^{+}, 0 \leq h^{\prime}(s) \leq \xi(s)\right\}
$$

and $\mathcal{N}_{h}$ is the null set where $h^{\prime}$ is not defined. Furthermore, if we denote $Q_{n}$ $:=\mathbb{R}^{+} \backslash \mathcal{A}_{n}$, then $\lim _{n \rightarrow \rightarrow \infty} \hat{h}\left(Q_{n}\right)=\hat{h}\left(Q_{h}\right)$ because $Q_{n+1} \subset Q_{n}$ for all $n$ and $\bigcap_{n} \mathcal{F}_{n}=Q_{h} \cup \mathcal{N}_{h}$. We take $\mathcal{A}_{t}=\widetilde{\mathcal{A}}_{n t}$ and $Q_{t}=\widetilde{Q}_{n t}$ in (3.21), we choose $\lambda_{1}=\lambda_{2}\left(h_{*}-\varepsilon\right)$ for small $\varepsilon<h_{*}$, then select $\lambda_{2}$

$$
\lambda_{2} \leq E I \eta_{4} /\left(L^{3}\left(\rho L+M_{s}\right) B V[h]\right),
$$

so that

$$
\frac{E I}{2}-\lambda_{2} \frac{L^{3}\left(\rho L+M_{s}\right)}{4 \eta_{4}} B V[h] \geq \frac{E I}{4},
$$

we obtain for all $t \geq t_{*}>0$

$$
\begin{gathered}
\frac{d}{d t} V(t) \leq \lambda_{2}\left(\eta_{4}-\varepsilon\right)\left(\rho\left\|w_{t}\right\|_{2}^{2}+M_{s} w_{t}^{2}(L, t)\right)+\left[\lambda_{3} \kappa+\lambda_{4} \int_{0}^{\infty} \xi(\sigma) d \sigma\right. \\
\left.\quad+B_{1}-\lambda_{2}\left(h_{*}-\varepsilon\right) E I\left(1-\frac{\kappa}{2}\right)\right]\left\|w_{x x}\right\|_{2}^{2}+\left(\frac{E I\left(h_{*}-\varepsilon\right)}{2} \lambda_{2}-\lambda_{3} \eta_{5}\right)
\end{gathered}
$$




$$
\begin{aligned}
& \times \int_{0}^{t} h(t-s)\left\|w_{x x}(s)\right\|_{2}^{2} d s+\left\{\lambda_{2}\left[E I\left(1+\frac{1-h_{*}}{4 \eta_{1}}+\frac{1}{\eta_{2}}\right)+\frac{T L}{2 \eta_{3}}\right] \kappa-\frac{E I}{4 n}\right\} \\
& \times \int_{0}^{L} \int_{\widetilde{\mathcal{A}}_{n t}} h(t-s)\left(w_{x x}(s)-w_{x x}(t)\right)^{2} d s d x+\lambda_{2}\left[\eta_{3}-\left(h_{*}-\varepsilon\right)\right] T\left\|w_{x}\right\|_{2}^{2} \\
+ & \lambda_{2}\left\{\left[\left(1+\eta_{2}\right)+\frac{L}{2 E I \eta_{3}}\right]\left(\int_{\widetilde{Q}_{n t}} h(t-s) d s\right)-\frac{h_{*}-\varepsilon}{2}\right\} E I\left(h \square w_{x x}\right) \\
+ & \left\{\frac{3}{4}\left[E I+\lambda_{2} \frac{L^{3}\left(\rho L+M_{s}\right)}{2 \eta_{4}} \int_{\widetilde{Q}_{n t}} \xi(t-\sigma) d \sigma\right]-\lambda_{4} \eta_{6}\right\} \int_{0}^{t} \xi(t-s) \\
& \times\left\|w_{x x}(s)\right\|_{2}^{2} d s-\lambda_{3}\left(1-\eta_{5}\right) \mu_{1}(t) V_{3}(t)-\lambda_{4}\left(1-\eta_{6}\right) \mu_{2}(t) V_{4}(t), \quad(3.22)
\end{aligned}
$$

where we have used

$$
\begin{aligned}
\int_{0}^{L} & \int_{\widetilde{Q}_{n t}} \xi(t-s)\left(w_{x x}(s)-w_{x x}(t)\right)^{2} d s d x \\
& \leq \frac{3}{2}\left(\int_{\widetilde{Q}_{n t}} \xi(t-s) d s\right)\left\|w_{x x}\right\|_{2}^{2}+\frac{3}{2} \int_{0}^{t} \xi(t-s)\left\|w_{x x}(s)\right\|_{2}^{2} d s \\
B_{1}= & \frac{3}{4}\left[E I+\lambda_{2} \frac{L^{3}\left(\rho L+M_{s}\right)}{2 \eta_{4}} \int_{\widetilde{Q}_{n t}} \xi(t-s) d s\right]\left(\int_{\widetilde{Q}_{n t}} \xi(t-s) d s\right) \\
& +\lambda_{2} E I\left(1-h_{*}\right)\left(\eta_{1}+\frac{3}{2} \int_{\widetilde{Q}_{n t}} h(t-s) d s\right) .
\end{aligned}
$$

Now, we start selecting the different parameters so that all the coefficients in the right-hand side of (3.22) be negative. First, we select $\eta_{1}$ and $\eta_{2}$ small enough and we choose $\eta_{3}=\left(g_{*}-\varepsilon\right) / 2$ and $\eta_{4}=\varepsilon / 2$. If $\mathcal{R}_{h}$ is sufficiently small, we have

$$
\begin{aligned}
& {\left[\left(1+\eta_{2}\right)+\frac{L}{2 E I \eta_{3}}\right]\left(\int_{\widetilde{Q}_{n t}} h(t-s) d s\right)-\frac{h_{*}-\varepsilon}{2}<0,} \\
& \frac{3\left(1-h_{*}\right)}{2}\left(\int_{\widetilde{Q}_{n t}} h(t-s) d s\right)<\sigma h_{*}\left(1-\frac{\kappa}{2}\right),
\end{aligned}
$$

with

$$
\sigma=\frac{3\left(1-h_{*}\right) \kappa}{4(2-\kappa) h_{*}}+\beta
$$

for a small $\beta>0$, large $n$ and $h_{*}>3 \kappa /(8-\kappa)$. Moreover we need to select $\lambda_{3}$, so that

$$
\frac{1-\varepsilon}{2} E I \lambda_{2} \leq \lambda_{3} \eta_{4} \leq \frac{(1-\delta)\left(h_{*}-\varepsilon\right)}{2 \kappa}(2-\kappa) E I \lambda_{2} \eta_{4} .
$$

Once $\eta_{1}, \eta_{2}, n$ and $t_{*}$ are fixed, we select also $\lambda_{2}$ such that

$$
\lambda_{2}\left[\left(1+\frac{1-h_{*}}{4 \eta_{1}}+\frac{1}{\eta_{2}}\right)+\frac{T L}{2 \eta_{3} E I}\right] \kappa-\frac{1}{4 n}<0 .
$$

Furthermore, we select $\lambda_{4}$ large enough so that

$$
\lambda_{4}>\frac{3}{4 \eta_{6}}\left[E I+\lambda_{2} \frac{L^{3}\left(\rho L+M_{s}\right)}{2 \eta_{4}} \int_{0}^{\infty} \xi(\sigma) d \sigma\right] .
$$


We need to take $\int_{0}^{\infty} \xi(s) d s$ small enough to get negative coefficients in the right-hand side of (3.22). Therefore,

$$
\frac{d}{d t} V(t) \leq-C_{1} \mathcal{E}(t)-C_{2} \mu_{1}(t) V_{3}(t)-C_{3} \mu_{2}(t) V_{4}(t)
$$

for some positive constants $C_{i}, i=1,2,3$.

If $\lim _{t \rightarrow \infty} \mu_{1}(t)=0$ or $\lim _{t \rightarrow \infty} \mu_{2}(t)=0$ then there exist $\bar{t} \geq t_{*}$ such that for all $t \geq \bar{t}$ we have

$$
\min \left\{C_{2} \mu_{1}(t), C_{3} \mu_{2}(t)\right\} \leq C_{1}
$$

Therefore, by the Lemma 3, we obtain

$$
\frac{d}{d t} V(t) \leq-\min \left\{C_{2} \mu_{1}(t), C_{3} \mu_{2}(t)\right\} \frac{V(t)}{\rho_{2}}, t \geq \bar{t}
$$

Then

$$
V(t) \leq \delta \exp \left(-\frac{1}{\rho_{2}} \int_{\bar{t}}^{t} \min \left\{C_{2} \mu_{1}(s), C_{3} \mu_{2}(s)\right\} d s\right), \quad t \geq \bar{t}, \quad \delta \geq 0 .
$$

By continuity and Lemma 3 again, it is easy to see

$$
\mathcal{E}(t) \leq \Lambda \exp \left(-\nu \int_{\bar{t}}^{t} \min \left\{\mu_{1}(s), \mu_{2}(s)\right\} d s\right), \quad t \geq 0
$$

for some positive constants $\Lambda$ and $\nu$.

If $\lim _{t \rightarrow \infty} \mu_{1}(t) \neq 0$ and $\lim _{t \rightarrow \infty} \mu_{2}(t) \neq 0$, then there exist a $\hat{t} \geq t_{*}$ and $C_{4}>0$ such that $\mu_{1}(t), \mu_{2}(t) \geq C_{4}$ for $t \geq \hat{t}$. Therefore

$$
\frac{d}{d t} V(t) \leq-C_{5}\left(\mathcal{E}(t)+V_{3}(t)+V_{4}(t)\right) \leq-\frac{C_{5}}{\rho_{2}} V(t), \quad C_{5}>0
$$

which leads to

$$
\mathcal{E}(t) \leq \Lambda e^{-\nu t}, t \geq \hat{t}
$$

for some positive constants $\Lambda$ and $\nu$. By continuity, we obtain a similar estimation on the interval $[0, \max \{\bar{t}, \hat{t}\}]$.

\section{Numerical simulations}

In this section, simulations for the presented system described by (1.1) and (1.2) are carried out to illustrate the effectiveness of the proposed control defined in (3.1). The corresponding system parameters are given in the Table 1 and, the initial displacement and velocity are $w_{0}(x)=\sin \left(\frac{\pi}{L} x\right)$ and $w_{1}(x)=0$, respectively. The kernel function is chosen to be $h(t)=e^{-k t}$, for some $k \in \mathbb{R}^{+}$.

We have used finite difference method for numerical approximation of the problem (1.1)-(1.2). Central difference approximations for both spatial and temporal derivatives, in the differential equation (1.1) and the boundary condition (1.2) have been adopted. For the convolution term, we have used the 
Table 1. The corresponding system parameters.

\begin{tabular}{lcr}
\hline Parameter & Description & Value \\
\hline$L$ & Length & $1000.00 \mathrm{~m}$ \\
$\rho$ & The uniform mass per unit length & $500 \mathrm{~kg} / \mathrm{m}$ \\
$E I$ & Bending stiffness & $1.5 \times 10^{7} \mathrm{Nm}$ \\
$T$ & Tension & $8.11 \times 10^{7} \mathrm{~N}$ \\
$M_{s}$ & Mass of the surface vessel & $9.60 \times 10^{6} \mathrm{~kg}$ \\
$d_{s}$ & Vessel damping & $1 \times 10^{3} \mathrm{Ns} / \mathrm{m}$ \\
\hline
\end{tabular}

Trapezoid method. We note that, the convolution term requires to store the solution from $t=0$ and use them at each time step. This, together with large values of the model parameters, demand very small values of time steps, that increases the computational time. Therefore, one can scale the parameters for computer simulations to overcome the time performance. The behavior of the solution of the model problem (1.1)-(1.2), with the control (3.1), have been illustrated in Figure 1 at $x=500,800$ and $x=1000 \mathrm{~m}$, respectively.

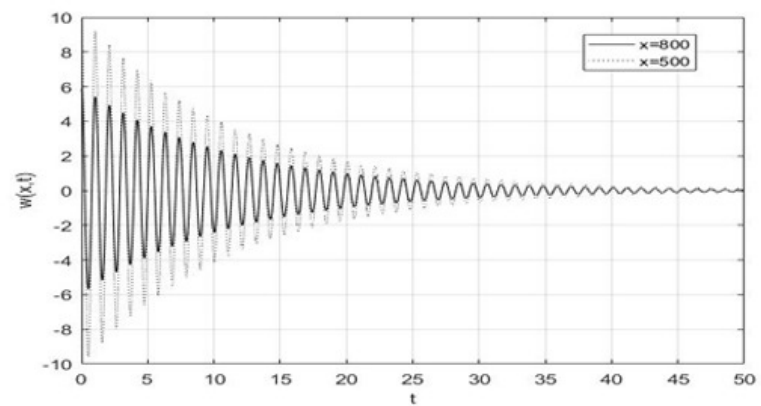

a)

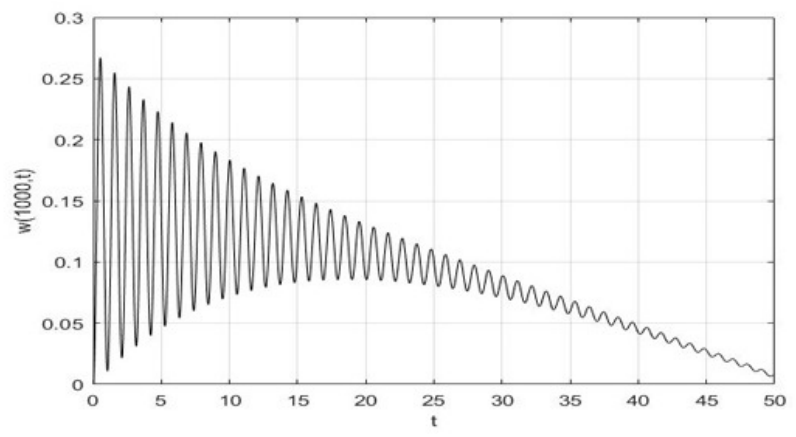

b)

Figure 1. Riser displacement at a) $x=500$ and $x=800$, b) $x=1000$.

In Figure 2, the discrepancy between the solutions with and without the control affect has been shown at three points $x=500,800$ and the boundary $x=L$. The affect of the control (3.1) can be seen, after a while due to the 


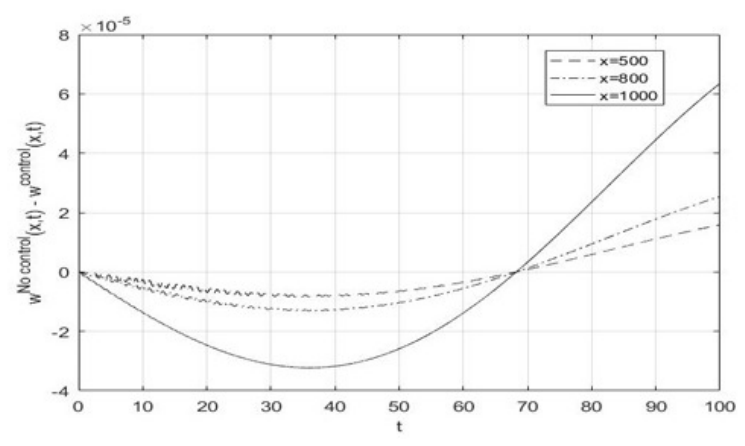

Figure 2. Difference between the riser displacement with/without control, at $x=500,800,1000 m$.

convolution term, in particular, when getting closer to the boundary point $x=L$.

\section{Conclusions}

In this paper, the vibration control of a viscoelastic flexible marine riser with vessel dynamics is studied. A boundary control has been designed to attenuate the vibrations of our system. With the proposed control, closed-looped stability for a large class of relaxation functions has been proven using the multiplier technique. The efficiency of the proposed boundary control is confirmed by some numerical simulations. In a future work, we intend to study the effect of different disturbances, such as the environmental disturbance on the vessel due to the ocean wave, wind and current.

\section{Acknowledgement}

The third author would like to thank King Fahd University of Petroleum and minerals for its continuous financial support.

\section{References}

[1] K.T. Andrews and M. Shillor. Vibrations of a beam with a damping tip body. Math. Comput. Model., 35(9-10):1033-1042, 2002. https://doi.org/10.1016/S0895-7177(02)00068-7.

[2] A. Berkani, N.-E. Tatar and A. Kelleche. Vibration control of a viscoelastic translational Euler-Bernoulli beam. J. Dyn. Control Syst., 24(1):167-199, 2017. https://doi.org/10.1007/s10883-017-9364-9.

[3] A. Berkani, N.-E. Tatar and A. Khemmoudj. Control of a viscoelastic translational Euler-Bernoulli beam. Math. Methods Appl. Sci., 40(1):237-254, 2017. https://doi.org/10.1002/mma.3985.

[4] F.C.L. Borges, N. Roitman, C. Magluta, D. A . Castello and R. Franciss. A concept to reduce vibrations in steel catenary risers by 
the use of viscoelastic materials. Ocean Engineering., 77:1-11, 2014. https://doi.org/10.1016/j.oceaneng.2013.12.004.

[5] M.S. de Querioz, D.M. Dawson, S.P. Nagarkatti and F. Zhang. Lyapunov-based control of mechanical system. Birkhauser, Boston, 2000. https://doi.org/10.1007/978-1-4612-1352-9.

[6] K.D. Do. Global stabilization of three-dimensional flexible marine risers by boundary control. Ocean Systems Eng., 1(2):171-194, 2011. https://doi.org/10.12989/ose.2011.1.2.171.

[7] M. Fabrizio and A. Morro. Mathematical Problems in Linear Viscoelasticity. SIAM Stud. Appl. Math. Philadelphia, 1992. https://doi.org/10.1137/1.9781611970807.

[8] S.S. Ge, W. He, B.V.E. How and Y.S. Choo. Boundary control of a coupled nonlinear flexible marine riser. Trans. Control Syst. Tech., 18(5):1080-1091, 2010. https://doi.org/10.1109/TCST.2009.2033574.

[9] B.Z. Guo and W. Guo. Adaptive stabilization for a Kirchhoff-type nonlinear beam under boundary output feedback control. Nonlinear Anal., 66(2):427-441, 2007. https://doi.org/10.1016/j.na.2005.11.037.

[10] F. Guo and F. Huang. Boundary feedback stabilization of the undamped EulerBernoulli beam with both ends free. SIAM J. Control Optim., 43(1):341-356, 2004. https://doi.org/10.1137/S0363012901380961.

[11] W. He and S.S. Ge. Cooperative control of a nonuniform gantry crane with constrained tension. Automatica, 66:146-154, 2016. https://doi.org/10.1016/j.automatica.2015.12.026.

[12] W. He, S.S. Ge, B.V.E. How, Y.S. Choo and K.S. Hong. Robust adaptive boundary control of a flexible marine riser with vessel dynamics. Automatica, 47(4):722-732, 2011. https://doi.org/10.1016/j.automatica.2011.01.064.

[13] W. He, S.S. Ge and S. Zhang. Adaptive boundary control of a flexible marine installation system. Automatica, 47(12):2728-2734, 2011. https://doi.org/10.1016/j.automatica.2011.09.025.

[14] W. He, Y. Ouyang and J. Hong. Vibration control of a flexible robotic manipulator in the presence of input deadzone. IEEE Trans. Appl. Ind. Informatics, 13(1):48-59, 2017. https://doi.org/10.1109/TII.2016.2608739.

[15] W. He and X. He ; C. Sun. Vibration control of an industrial moving strip in the presence of input deadzone. IEEE Trans. Ind. Electron, 64(6):4680-4689, 2017. https://doi.org/10.1109/TIE.2017.2674592.

[16] W. He and S. Zhang. Control design for nonlinear flexible wings of a robotic aircraft. IEEE Trans. Control Syst. Technol., 25(1):351-357, 2017. https://doi.org/10.1109/TCST.2016.2536708.

[17] B.V.E. How, S.S. Ge and Y.S. Choo. Active control of flexible marine risers. J. Sound Vib., 320(4-5):758-776, 2009. https://doi.org/10.1016/j.jsv.2008.09.011.

[18] Y.H. Kang, J.Y. Park and J.A. Kim. A memory type boundary stabilization for an Euler-Bernoulli beam under boundary output feedback control. J. Korean Math. Soc., 49(5):947-964, 2012. https://doi.org/10.4134/JKMS.2012.49.5.947.

[19] A. Kelleche, A. Berkani and N.-E. Tatar. Uniform stabilization of a nonlinear axially moving string by a boundary control of memory type. J. Dyn. Control Syst., pp. 1-11, 2017. https://doi.org/10.1007/s10883-017-9370-y. 
[20] A.M. Krall. Asymptotic stability of the Euler-Bernoulli beam with boundary control. J. Math Anal. Appl., 137(1):288-295, 1989. https://doi.org/10.1016/0022247X(89)90289-8.

[21] S. Li, Y. Wang and Z. Liang. Stabilization of vibrating beam with a tip mass controlled by combined feedback forces. J. Math. Anal. Appl., 256(1):13-38, 2001. https://doi.org/10.1006/jmaa.2000.7217.

[22] Y. Liu and F. Guo. Output feedback boundary control of a flexible marine riser system. J. Vib. Control, 2017. https://doi.org/10.1177/1077546317708516.

[23] Y. Liu, H. Huang, H. Gao and X. Wu. Modeling and boundary control of a flexible marine riser coupled with internal fluid dynamics. J. Control Theory Appl., 11(2):316-323, 2013. https://doi.org/10.1007/s11768-013-1245-5.

[24] Y. Liu, Z. Zhao and W. He. Boundary control of an axially moving accelerated/decelerated belt system. Int. J. Robust Nonlinear Control, 26(17):38493866, 2016. https://doi.org/10.1002/rnc.3538.

[25] Y. Liu, Z. Zhao and W. He. Stabilization of an axially moving accelerated/decelerated system via an adaptive boundary control. ISA Transactions, 64:394-404, 2016. https://doi.org/10.1016/j.isatra.2016.04.006.

[26] Y. Liu, Z. Zhao and W. He. Boundary control of an axially moving system with high acceleration/deceleration and disturbance observer. J. Franklin Inst., 354(7):2905-2923, 2017. https://doi.org/10.1016/j.jfranklin.2017.01.026.

[27] J.Y. Park, Y.H. Kang and J.A. Kim. Existence and exponential stability for a Euler-Bernoulli beam equation with memory and boundary output feedback control term. Acta. Appl. Math., 104(3):287-301, 2008. https://doi.org/10.1007/s10440-008-9257-8.

[28] J.Y. Park and J.A. Kim. Existence and uniform decay for EulerBernoulli beam equation with memory term. Math. Meth. Appl. Sci., 27(14):1629-1640, 2004. https://doi.org/10.1002/mma.512.

[29] L. Seghour, A. Khemmoudj and N.-E. Tatar. Control of a riser through the dynamic of a vessel. Appl. Anal., 95(9):1957-1973, 2016. https://doi.org/10.1080/00036811.2015.1080249.

[30] N.-E. Tatar. Uniform decay in viscoelasticity for kernels with small non-decreasingness zones. Appl. Math. Comput., 218(15):7939-7946, 2012. https://doi.org/10.1016/j.amc.2012.02.012. 